OPEN ACCESS

Edited by:

Yi-Cheng Sun,

Chinese Academy of Medical

Sciences and Peking Union Medical

College, China

Reviewed by:

Qian Guoliang,

Nanjing Agricultural University, China

Hongxia Wang,

University of Alabama at Birmingham,

United States

*Correspondence:

Pinglan L

lipinglan420@126.com

Nan Shang

nshang@ualberta.ca

Specialty section:

This article was submitted to

Food Microbiology,

a section of the journal

Frontiers in Microbiology

Received: 27 April 2017 Accepted: 12 September 2017

Published: 26 September 2017

Citation:

Liu L, Wu R, Zhang J, Shang N and

Li P (2017) D-Ribose Interferes with

Quorum Sensing to Inhibit Biofilm

Formation of Lactobacillus paraplantarum L-ZS9.

Front. Microbiol. 8:1860.

doi: 10.3389/fmicb.2017.01860

\section{D-Ribose Interferes with Quorum Sensing to Inhibit Biofilm Formation of Lactobacillus paraplantarum L-ZS9}

\author{
Lei Liu ${ }^{1,2}$, Ruiyun $W u^{1}$, Jinlan Zhang ${ }^{1}$, Nan Shang ${ }^{1,3 *}$ and Pinglan $L^{1,2 *}$ \\ ${ }^{1}$ Beijing Advanced Innovation Center for Food Nutrition and Human Health, College of Food Science and Nutritional \\ Engineering, China Agricultural University, Beijing, China, ${ }^{2}$ Key Laboratory of Functional Dairy, China Agricultural University, \\ Beijing, China, ${ }^{3}$ Department of Agricultural, Food and Nutritional Sciences, University of Alberta, Edmonton, AB, Canada
}

Biofilms help bacteria survive under adverse conditions, and the quorum sensing (QS) system plays an important role in regulating their activities. Quorum sensing inhibitors (QSIs) have great potential to inhibit pathogenic biofilm formation and are considered possible replacements for antibiotics; however, further investigation is required to understand the mechanisms of action of QSIs and to avoid inhibitory effects on beneficial bacteria. Lactobacillus paraplantarum L-ZS9, isolated from fermented sausage, is a bacteriocin-producing bacteria that shows potential to be a probiotic starter. Since exogenous autoinducer-2 (Al-2) promoted biofilm formation of the strain, expression of genes involved in Al-2 production was determined in L. paraplantarum L-ZS9, especially the key gene luxS. D-Ribose was used to inhibit biofilm formation because of its Al-2 inhibitory activity. Twenty-seven differentially expressed proteins were identified by comparative proteomic analysis following D-ribose treatment and were functionally classified into six groups. Real-time quantitative PCR showed that Al-2 had a counteractive effect on transcription of the genes tuf, fba, gap, pgm, nfo, rib, and rpoN. Over-expression of the tuf, fba, gap, pgm, and rpoN genes promoted biofilm formation of $L$. paraplantarum L-ZS9, while over-expression of the nfo and rib genes inhibited biofilm formation. In conclusion, D-ribose inhibited biofilm formation of $L$. paraplantarum L-ZS9 by regulating multiple genes involved in the glycolytic pathway, extracellular DNA degradation and transcription, and translation. This research provides a new mechanism of QSI regulation of biofilm formation of Lactobacillus and offers a valuable reference for QSI application in the future.

Keywords: Lactobacillus paraplantarum L-ZS9, quorum sensing inhibitor, biofilms, proteomics, recombinant strains

\section{INTRODUCTION}

Bacteria live in dense and diverse communities termed biofilms, which is a major mode of microbial life (Nadell et al., 2016). Biofilms are sessile microbial communities that attach to biotic and abiotic surfaces and survive as self-organized, three-dimensional structures by producing an extracellular polymeric matrix (Penesyan et al., 2015). This lifestyle helps microorganisms to survive in unfavorable environments (Taylor et al., 2014; Olsen, 2015). Biofilms of pathogenic bacteria may cause antibiotic-tolerant infections as well as damage to surfaces and flow systems (Hoiby et al., 2010; Bixler and Bhushan, 2012; Drescher et al., 2013). However, similar to some non-pathogenic 
microorganisms and beneficial bacteria, biofilms enable pathogenic bacteria to resist environmental conditions, leading to successful colonization and maintenance of their population (Lepargneur and Rousseau, 2002).

Within biofilms, bacteria use a quorum sensing (QS) system to communicate with each other. The LuxS/AI-2 QS system exists widely in both Gram-negative and Gram-positive bacteria. The signal molecule, autoinducer-2 (AI-2), has been deemed as a universal language for intra-species and inter-species communication (Schauder et al., 2001; Gospodarek et al., 2009). The luxS AI-2 synthase gene homolog has been found in a wide range of bacteria, with $17 \%$ of the phylum Bacteroidetes and $83 \%$ of the Firmicutes predicted to have the homolog according to the KEGG database (Thompson et al., 2015). Although most research has focused on the regulation of AI-2 in biofilms of pathogens, some non-pathogenic and beneficial bacteria have been reported to use this 'universal' and 'common' signaling system to regulate their behavior (Park et al., 2016). For example, AI-2 signaling has been reported to regulate cell growth and metabolism of Lactobacillus (Buck et al., 2009; Moslehi-Jenabian et al., 2009; Park et al., 2016). And in Bifidobacteria, AI-2 activity correlates with biofilm formation and gut colonization (Christiaen et al., 2014; Sun et al., 2014).

Since QS inhibitors (QSIs) hinder biofilm formation and reduce bacterial virulence in the biofilm state, they are considered as an ideal tool to inhibit the biofilm formation of pathogens and provide a good target to control bacterial infection (Pan and Ren, 2009; Harjai et al., 2014). An ideal QSI should significantly interfere with the QS system and social behavior without toxic effects on the bacteria and/or host. Ribose has no toxic effects and shares structural similarity with AI-2, which exhibits a furanosyl borate diether form (Cao and Meighen, 1989). This resemblance has been thought to cause competition between AI-2 and ribose. Thus, ribose has been used as a QSI to inhibit biofilm formation of various pathogenic bacteria (Armbruster et al., 2011; Lee et al., 2015; Cho et al., 2016; Ryu et al., 2016). However, the underlying mechanism of biofilm inhibition of non-pathogenic and beneficial bacteria still needs to be investigated.

Recently, LuxS/AI-2 QSIs have shown great potential to replace antibiotics to control pathogenic biofilm formation and infection (Brackman et al., 2009, 2011; Roy et al., 2013; Ryu et al., 2016). However, adequate considerations should be noted when QS therapy develops rapidly. Because of the common existence of LuxS/AI-2 QS in microorganisms, LuxS/AI-2 QSIs may inhibit biofilm formation of non-pathogenic and beneficial organisms as well. Lactobacillus paraplantarum L-ZS9 was originally isolated from fermented sausage and was proven to produce class IIb bacteriocins to inhibit the growth of pathogenic bacteria in our previous study (Wang et al., 2015; Zhang et al., 2016). It has the potential to be used as a probiotic starter. This study determined AI-2 production in L-ZS9 and AI-2 inhibitory activity by D-ribose. The effects of D-ribose on biofilm formation of L-ZS9 and its underlying mechanism were investigated and analyzed. This study provides new information about the regulation mechanism of this QSI on biofilm formation of Lactobacillus and has value as a reference for the reasonable application of QSI.

\section{MATERIALS AND METHODS}

\section{Bacterial Strains and Culture}

Lactobacillus paraplantarum L-ZS9 was incubated at $37^{\circ} \mathrm{C}$ in de Man-Rogosa-Sharpe (MRS) broth (Bridge, Beijing) and MRS agar aerobically. Escherichia coli DH5 $\alpha$ (Takara, Dalian) was incubated in Lennox broth or on solid medium with $1.5 \%(\mathrm{w} / \mathrm{v})$ agar at $37^{\circ} \mathrm{C}$. DH5 $\alpha$ and L-ZS9 transformed with pMG76e vector were cultured with 200 and $3 \mu \mathrm{g} / \mathrm{mL}$ erythromycin, respectively. The pMG76e vector was provided by Professor Shangwu Chen from China Agricultural University (CAU, Beijing, China). Vibrio harveyi BB170 and BB152 were provided by Professor Xiangan Han from Shanghai Veterinary Research Institute (CAAS, Shanghai, China) and cultured in Marine Broth 2216 (Difco, United States) or Autoinducer Bioassay (AB) medium (Bassler et al., 1993).

\section{Analysis of Genes Related to Al-2 Production}

The complete genome sequence of L. paraplantarum L-ZS9 is deposited in GenBank with accession number CP013130 (Liu and Li, 2016). The $p f s, \operatorname{luxS}$, metE/met $H$, and metK genes involved in AI-2 production and the $s a h H$ gene responsible for metabolism of $S$-adenosylhomocysteine $(\mathrm{SAH})$ to $S$-ribosylhomocysteine in one-step were searched for in the genome sequence of L. paraplantarum L-ZS9 according to their gene annotations by using the BLAST program of NCBI. Sizes of these genes were analyzed by Primer Input $3.0^{1}$, and locations were determined by analyzing the genome map.

\section{Growth-Curve Assay}

To determine the effects of AI-2 and D-ribose on the growth of L. paraplantarum L-ZS9, a growth-curve assay was conducted. L. paraplantarum L-ZS9 was cultured in MRS broth in the presence or absence of AI-2 (3.7, 18.5, 37, $185 \mu \mathrm{M})$ (initial concentration $3.7 \mathrm{mM}$, purchased from OMM Scientific, United States). L. paraplantarum L-ZS9 was also cultured in MRS broth in the presence or absence of D-ribose $(0,10,50$, and $100 \mathrm{mM}$ ). Cultures were incubated at $37^{\circ} \mathrm{C}$ for $36 \mathrm{~h}$. After 3, 6, 9, $12,15,18,21,24,27,30,33$, and $36 \mathrm{~h}$, the optical density at $600 \mathrm{~nm}$ was determined using a UV-1800 Spectrophotometer (Shanghai Meipuda instrument, China).

\section{Determination of Al-2-Mediated Bioluminescence and Al-2 Inhibitory Activity of D-Ribose}

Lactobacillus paraplantarum L-ZS9 was cultured in 12\% (w/v) skim milk medium. After culture for 2, 4, 6, 8, 10, 12, 14, 16, 18, 20,22 , and $24 \mathrm{~h}$, cells were collected by centrifuging at $12,000 \mathrm{~g}$, $4^{\circ} \mathrm{C}$, for $10 \mathrm{~min}$. The cell-free culture fluid (CF) was obtained by filtering through a $0.22-\mu \mathrm{m}$ filter (Millipore, Bedford, MA, United States) and adjusted to $\mathrm{pH}$ 7.0. The reporter strain $V$. harveyi $\mathrm{BB} 170$ was diluted 1:5000 with $\mathrm{AB}$ medium, and the CF sample was added to the diluted BB170 culture at 1:10 (v/v).

\footnotetext{
${ }^{1}$ http://primer3.ut.ee/
} 
The mixture was incubated at $28^{\circ} \mathrm{C}$ for $5 \mathrm{~h}$, and $100 \mu \mathrm{L}$ aliquots were added to white, flat-bottomed, 96-well plates (Thermo Labsystems, Franklin, MA, United States) to detect AI-2 activity. The CF collected from BB152 and DH5 $\alpha$ cultures was used as a positive and negative control, respectively. Luminescence was measured using a Tecan GENios Plus microplate reader in luminescence mode (Tecan Austria GmbH, Grodig, Austria).

D-Ribose inhibition of BB152 and L-ZS9 AI-2 activity was also determined by using the $\mathrm{BB} 170$ reporter strain. D-Ribose $(0,10$, 50 , and $100 \mathrm{mM}$ ) was added to $\mathrm{AB}$ medium containing diluted BB170 culture $(1: 5000, v / v)$ with the CF of BB152 (1:10, v/v) or L-ZS9 $(1: 10, v / v)$. After incubation at $28^{\circ} \mathrm{C}$ for $5 \mathrm{~h}$, AI-2 activity was detected as described above.

\section{Biofilm Formation Assay}

Crystal violet $(\mathrm{CV})$ staining was used to quantify biofilm formation of L. paraplantarum L-ZS9. Briefly, overnight culture of L. paraplantarum L-ZS9 was diluted to an optical density of 0.1 at $600 \mathrm{~nm}$. Diluted culture $(200 \mu \mathrm{L})$ was transferred to a 96-well plate (Corning, United States). Different concentrations of AI-2 (3.7, 18.5, 37, $185 \mu \mathrm{M})$, D-ribose $(10,50$, and $100 \mathrm{mM})$ was added, and the mixture was incubated at $37^{\circ} \mathrm{C}$ for $36 \mathrm{~h}$. The wells were washed gently three times with phosphate-buffered saline, stained with $0.1 \% \mathrm{CV}$ for $30 \mathrm{~min}$ at room temperature, rinsed with distilled water, air-dried, and $100 \mu \mathrm{L}$ 95\% ethanol added to dissolve the CV. The absorbance at $595 \mathrm{~nm}$ was determined using a Synergy 2 microplate reader (Biotek, Winooski, VT, United States).

\section{Proteomic Analysis}

Lactobacillus paraplantarum L-ZS9 treated with and without D-ribose $(100 \mathrm{mM})$ was used for gel-based proteome analysis. Briefly, total-cell protein was extracted by trichloroacetic acid / acetone precipitation (Lakshman et al., 2008). Isolated protein was resuspended in lysis buffer ( $7 \mathrm{M}$ urea, $2 \mathrm{M}$ thiourea, $2 \%, w / v, C H A P S)$ and stored at $-80^{\circ} \mathrm{C}$ until analysis. Protein concentration was determined by Bradford protein assay (Bio-Rad).

For electrophoresis, $400 \mu \mathrm{g}$ of total protein was loaded onto immobilized $\mathrm{pH}$-gradient strips $(17 \mathrm{~cm}$, non-linear $\mathrm{pH}$ 4-7; Bio-Rad). Isoelectric focusing and sodium dodecyl sulfate polyacrylamide gel electrophoresis were carried out as previously described (Wang et al., 2006). After electrophoresis, gels were stained with Colloidal Coomassie Brilliant Blue G-250, imaged using an ImageScanner (Amersham Biosciences), and analyzed by ImageMaster 2D Platinum 6.0 software (Wang et al., 2006). Protein spots with $\geq 1.5$-fold change in abundance $(p<0.05)$ were subjected to protein identification.

The selected protein spots were excised, and in-gel digestion was performed with $0.01 \mu \mathrm{g} / \mu \mathrm{L}$ trypsin (Promega, Madison, WI, United States) in $25 \mathrm{mM}$ ammonium bicarbonate. Tryptic peptides were dissolved in $0.5 \%(\mathrm{w} / \mathrm{v})$ trifluoroacetic acid. Matrix-assisted laser desorption/ionization time-of-flight mass spectrometry and tandem time-of-flight/time-of-flight mass spectrometry were carried out on a 4800 Proteomics Analyzer (Applied Biosystems, United States) as described previously (Wang et al., 2006). Combined mass with mass/mass spectra was used to interrogate protein sequences in the NCBI database using the MASCOT database search algorithms (version 2.1, Matrix Science, London, United Kingdom). A protein was reported as identified if the Mascot score confidence interval > 95\%. Functional analyses of the differentially expressed proteins were conducted using the Universal Protein Resource ${ }^{2}$ and the Kyoto Encyclopedia of Genes and Genomes ${ }^{3}$.

\section{Quantitative Real-Time PCR (qRT-PCR)}

Lactobacillus paraplantarum L-ZS9 was cultured in MRS with D-ribose $(100 \mathrm{mM})$ or AI-2 $(18.5 \mu \mathrm{M})$ at $37^{\circ} \mathrm{C}$ to logarithmic phase. L. paraplantarum L-ZS9 cultured without AI-2 or D-ribose was used as a control. Total RNA was extracted with TRIzol (Invitrogen, United States) according to the manufacturer's instructions. RNA quality was determined by measuring $A_{260} / A_{280}$ and $A_{260} / A_{230}$ and by gel electrophoresis. Isolated RNA was transcribed into single-stranded cDNA using a TUREscript 1st Strand cDNA Synthesis Kit (Aidlab, China). qRTPCR was performed by using SYBR Green assay kit (Tiangen, China) and a 7500 Fast Real-Time PCR system (Applied Biosystems). Primers were designed by Primer 3 Input $^{4}$. The $16 S$ rRNA gene was used as an internal reference. The relative expression of specific genes was calculated by using the $2^{-\Delta \Delta C_{\mathrm{T}}}$ method according to Livak and Schmittgen (2001).

\section{Construction of Plasmids and Bacterial Strains}

Chromosomal DNA of L. paraplantarum L-ZS9 was isolated using a TIANamp Bacteria DNA Kit (Tiangen Biotech, Beijing). The genes tuf, fba, gap, pgm, nfo, rib, and rpoN were amplified from the chromosomal DNA by PCR and cloned into pMD18T vector (Takara, Dalian). The constructed tuf, fba, gap, pgm, $n f o$, rib, and rpoN-pMD18T vectors and the pMG76e vector (provided by Professor Shangwu Chen, China Agricultural University) were digested with Fastdigest enzymes XbaI and XhoI (Thermo, United States). The gene fragments were inserted into the linearized pMG76e plasmid using a Rapid DNA Ligation Kit (Thermo, United States), and the constructed vector was transformed into E. coli DH5 $\alpha$. The constructed plasmids harboring the genes of interest were identified by PCR and extracted by using a TIANpure Midi Plasmid Kit (Tiangen, China). Constructed pMG76e and empty pMG76e plasmids were electrotransformed into L. paraplantarum L-ZS9 competent cells, and recombinant strains were selected with erythromycin and confirmed by PCR.

\section{Biofilm Formation of Recombinant Strains}

The growth of recombinant strains was analyzed by measuring the optical density at $600 \mathrm{~nm}$ to determine the effect of the expression the genes of interest on the growth of L-ZS9. Biofilm formation of recombinant strains over-expressing tuf, fba, gap,

\footnotetext{
${ }^{2}$ http://www.ebi.ac.uk/uniprot/

${ }^{3}$ http://www.genome.jp/kegg/

${ }^{4}$ http://primer3.ut.ee/
} 
A

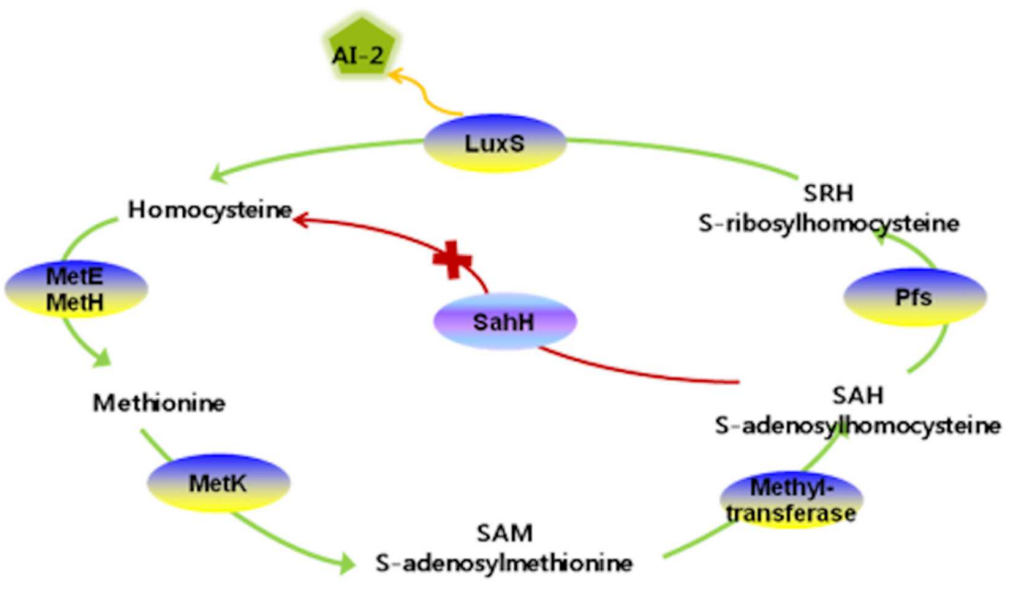

B

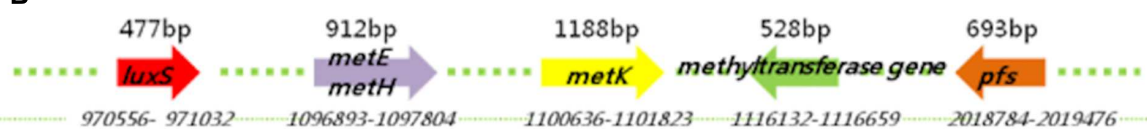

FIGURE 1 | Al-2 production pathway (A) and sizes and locations of related genes (B) in L. paraplantarum L-ZS9.

$p g m, n f o, r i b$, and $r p o N$ was measured by CV staining. Overnight cultures of recombinant strains and the wild-type strain were diluted in MRS without erythromycin to an optical density of 0.1 at $600 \mathrm{~nm}$, and $200 \mu \mathrm{L}$ of each suspension was transferred to a 96-well plate (Corning, NY, United States). After incubation at $37^{\circ} \mathrm{C}$ for $36 \mathrm{~h}$, the biofilm formation of these strains was measured as described above.

\section{Statistical Analysis}

The data were analyzed by one-way analysis of variance (ANOVA) using Graphpad Prism 5.0. Data are presented as means \pm SEM. A $p$-value less than 0.05 was considered as significant.

\section{RESULTS}

\section{L. paraplantarum L-ZS9 Contains All the Genes Responsible for Al-2 Production But Not sahH}

Biosynthesis of AI-2 is part of the methionine catabolism cycle. MetE/MetH is responsible for transforming homocysteine into methionine. Methionine is then converted to $S$-adenosylmethionine (SAM) in a reaction catalyzed by MetK. Methyltransferase transforms SAM to SAH, which is converted to homocysteine through Pfs and LuxS, and AI-2 is produced (Schauder et al., 2001; Parveen and Cornell, 2011). Complete genome analysis showed that L. paraplantarum L-ZS9 contains all the genes involved in AI-2 production, including $p f s$, luxS, metE/met $H$, metK, and the methyltransferase gene, but $s a h H$ or its homologous gene, responsible for changing $\mathrm{SAH}$ to homocysteine directly, was not found (Figure 1A). These results indicated that $L$. paraplantarum L-ZS9 metabolized SAH to homocysteine through Pfs and LuxS, and produced AI-2 as a byproduct. The size and location of these genes are shown in Figure 1B.

\section{L. paraplantarum L-ZS9 Produced AI-2 When Cultured in Skim Milk Medium}

AI-2 production was measured by using the reporter strain $V$. harveyi $\mathrm{BB} 170$ in a bioassay with CFs of L. paraplantarum

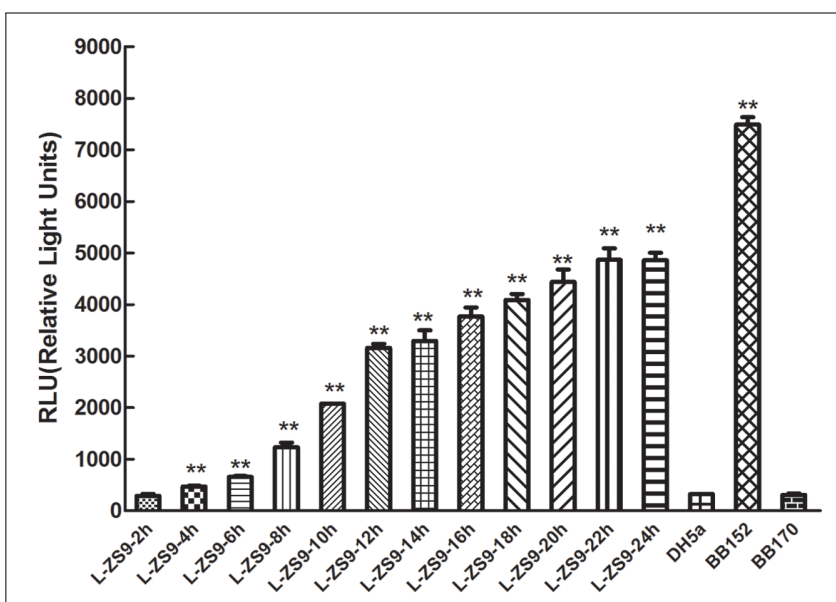

FIGURE 2 | Al-2 activity in cell-free culture fluids (CFs) of $L$. paraplantarum L-ZS9. V. harveyi BB152 served as a positive control and E. coli DH5 $\alpha$ as a negative control. Data are presented as mean \pm SEM. $n \geq 3$. ${ }^{*} p<0.05$,

${ }^{* *} p<0.01$ compared with the negative control. 

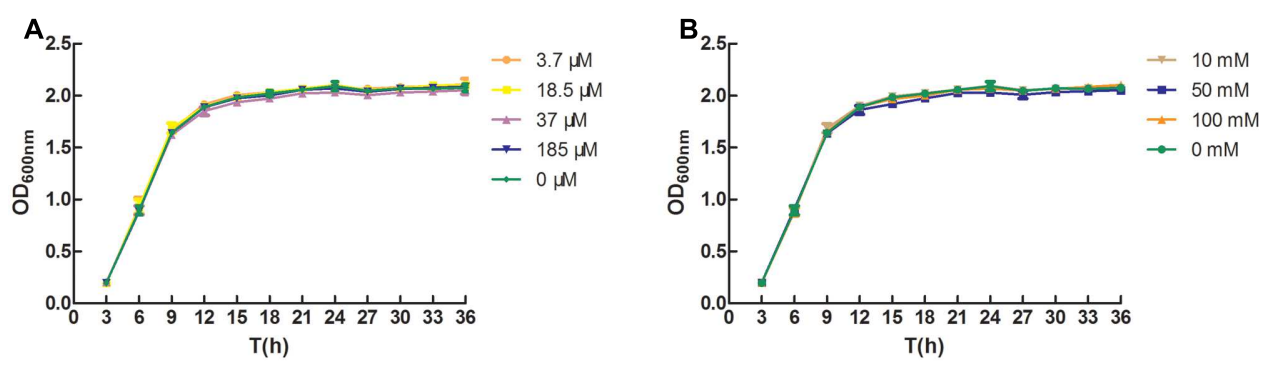

FIGURE 3 | Effects of Al-2 (A) and D-ribose (B) on growth of L. paraplantarum L-ZS9. Growth of the strain in the absence of Al-2 or D-ribose served as a control. Data are presented as mean \pm SEM. $n \geq 3$. ${ }^{*} p<0.05,{ }^{* *} p<0.01$ compared with the control.
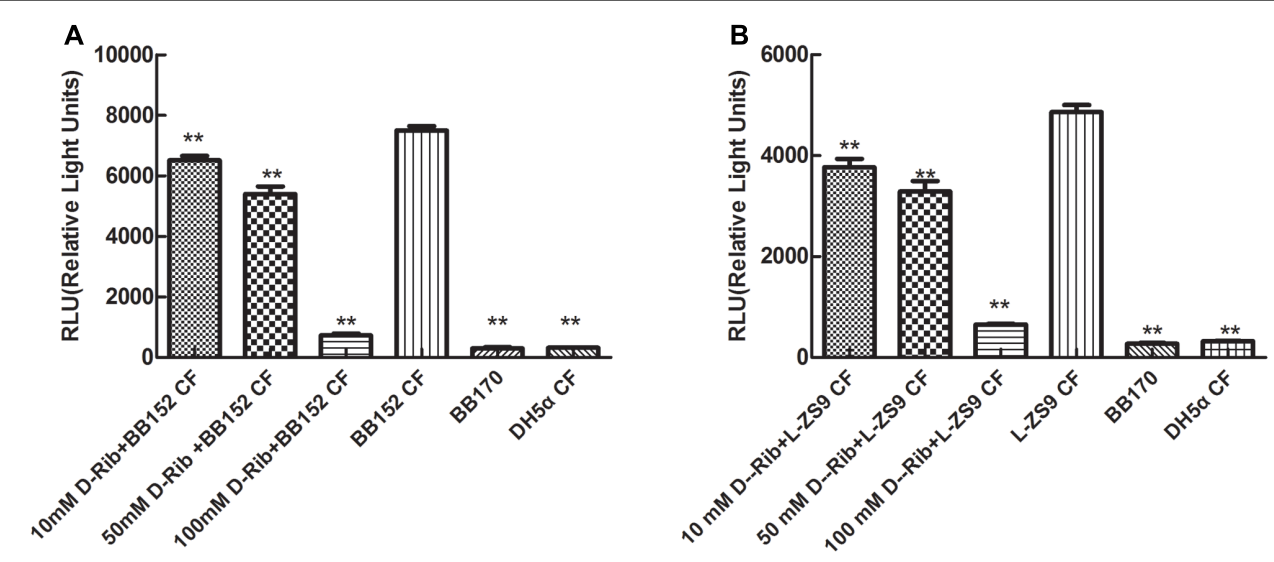

FIGURE 4 | Effect of D-ribose on Al-2 activity of V. harveyi BB152 (A) and L. paraplantarum L-ZS9 (B). E. coli DH5 $\alpha$ served as a negative control. Data are presented as mean \pm SEM. $n \geq 3 .{ }^{*} p<0.05,{ }^{* *} p<0.01$ compared with the positive control.
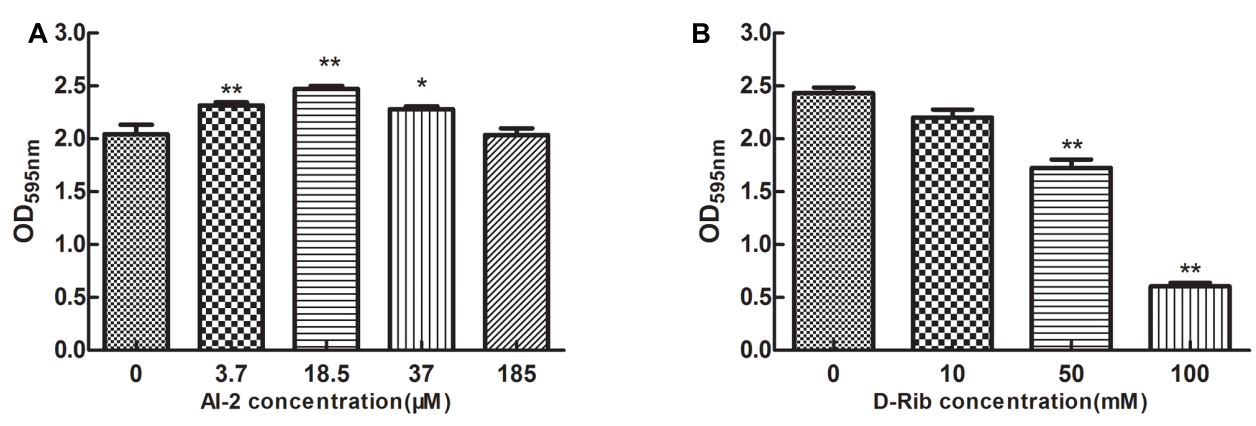

FIGURE 5 | Effect of Al-2 (A) and D-ribose (B) on biofilm formation of L. paraplantarum L-ZS9. The biofilm formation of the strain in the absence of Al-2 or D-ribose served as a control. Data are presented as mean \pm SEM. $n \geq 3 .{ }^{*} p<0.05,{ }^{* *} p<0.01$ compared with the control.

L-ZS9 cultured in skim milk medium. V. harveyi BB152 CF with confirmed AI-2 activity (7492.604 relative light units (RLUs)) was used as a positive control, E. coli DH5 $\alpha$ CF with no AI-2 activity (321.805 RLUs) was used as a negative control, and $V$. harveyi BB170 with no CF (307.740 RLUs) was used as a blank control. AI-2 activity was detected in CFs prepared from L. paraplantarum L-ZS9 grown in skim milk medium and increased with increasing incubation time to reach a maximum value (4864.748 RLUs) after $22 \mathrm{~h}$ (Figure 2). These results suggested that $L$. paraplantarum L-ZS9 can produce AI-2 and that the AI-2 concentration depended on the incubation time.

\section{Inhibitory Effect of D-Ribose on Al-2 Activity of BB152 and L-ZS9}

As shown in Figures 3A,B, exogenous AI-2 and D-ribose had no influence on growth of $L$. paraplantarum L-ZS9. D-Ribose significantly inhibited the AI-2 activity of $V$. harveyi BB152 and L. paraplantarum L-ZS9 in a dose-dependent manner (Figures 4A,B); $100 \mathrm{mM}$ D-ribose inhibited AI-2 activity 

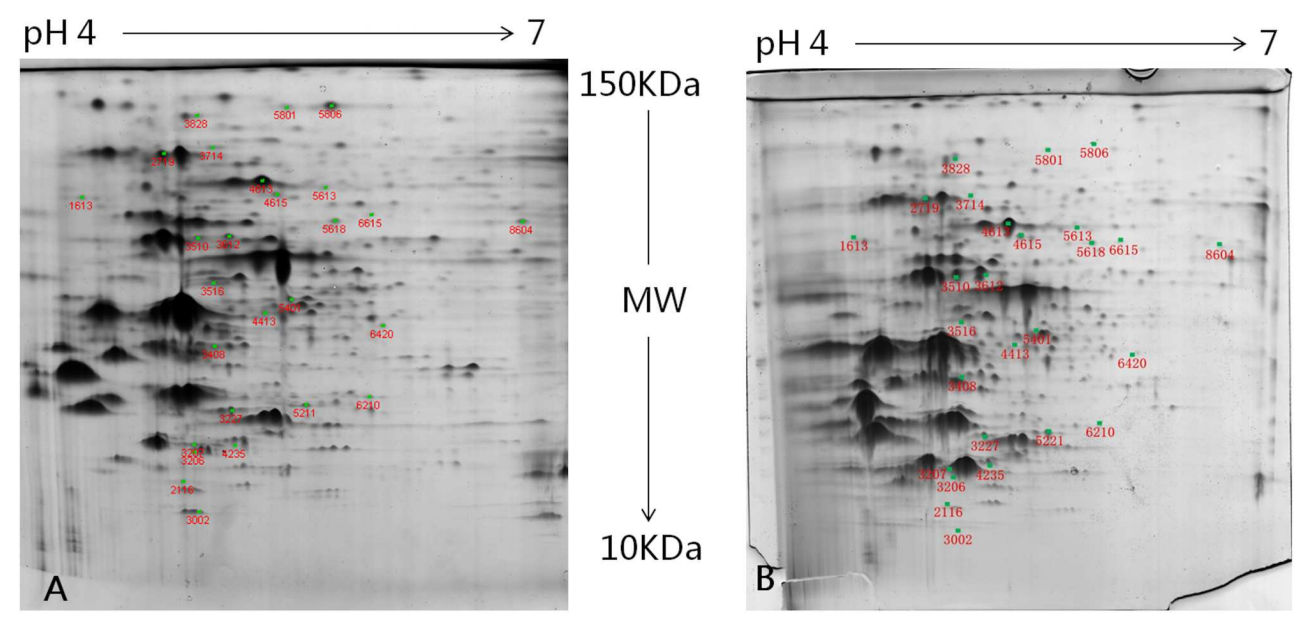

FIGURE 6 | Two-dimensional electrophoresis of the total-cell proteins of L. paraplantarum L-ZS9 treated without (A) or with D-ribose (B). Differentially expressed protein spots of cells treated with or without D-ribose were numbered according to the numbering in Table $\mathbf{1 .}$

of $V$. harveyi BB152 and L. paraplantarum L-ZS9 to about 0.10 -fold and 0.13 -fold, respectively. The result indicated that D-ribose could be used as a QSI against AI-2 activity of L-ZS9.

\section{Inhibitory Effect of D-Ribose on Biofilm Formation of L-ZS9 Induced by Al-2}

Figure 5A shows that 3.7-37.0 $\mu \mathrm{M}$ AI-2 increased biofilm formation of $L$. paraplantarum L-ZS9, which peaked at 18.5 $\mu \mathrm{M}$ AI-2. Different concentrations of AI-2 showed different effects on biofilm formation of L-ZS9. The effect of AI-2 on biofilm formation was not dose-dependent; the highest biofilm formation was observed when cultures were treated with $18.5 \mu \mathrm{M}$ AI-2, and decreased with increasing concentration of AI-2. On the contrary, D-ribose inhibited the biofilm formation of L-ZS9 in a dose-dependent manner, and $100 \mathrm{mM}$ D-ribose inhibited the biofilm formation of L-ZS9 to about 0.25 -fold compared with the control (Figure 5B).

\section{Protein Identification in L. paraplantarum L-ZS9 Treated with or without D-Ribose}

To profile the impact of D-ribose on protein expression, L. paraplantarum L-ZS9 was cultured with or without D-ribose. The proteomes were comparatively studied by gel-based proteomic analysis. Preliminary 2D gel electrophoresis analysis with a wide $\mathrm{pH}$ gradient $(\mathrm{pH} \mathrm{3-10)}$ showed that the majority of proteins had their isoelectric point (pI) in the acidic region (data not shown). Therefore an immobilized $\mathrm{pH}$-gradient strip with a $\mathrm{pH}$ gradient of 4-7 was used in this study. As shown in Figure 6, 27 differentially expressed proteins were detected $(P<0.05)$ (details of biological replicates are provided in the Supplementary Material). Among them, 15 proteins showed up-regulation and 12 proteins showed downregulation by at least 1.5 -fold following $\mathrm{D}$-ribose treatment. The molecular weight (MW) and $\mathrm{pI}$ of these proteins are listed in Table 1. Functionally, these differentially expressed proteins were classified into six groups that related to carbon and carbohydrate metabolism, amino acid metabolism, nucleotide transport and metabolism, fatty acid metabolism, transcription and translation, and other functions. Function classification of these proteins suggested that D-ribose may inhibit biofilm through multiple mechanisms, which include regulating translation and transcriptional activity, carbohydrate and energy metabolism, amino acid synthesis, and protein and enzyme secretion.

\section{Effects of D-Ribose and Al-2 on Gene Transcription of $L$. paraplantarum L-ZS9}

As shown in Figure 7, D-ribose changed the transcription of the tuf, fba, gap, pgm, nfo, rib, and rpoN genes of L. paraplantarum L-ZS9, which was consistent with the effect of D-ribose on their protein levels. These genes were selected from those encoding the differentially expressed proteins of the above $2 \mathrm{D}$ polyacrylamide gel electrophoresis results based on their relationship to QS, which has been reported in previous research (Wolfe et al., 2004; Da et al., 2008; Kiedrowski et al., 2011; Iyer and Hancock, 2012; Sahu et al., 2012; Zhou et al., 2012; Hao et al., 2013; Li Z. et al., 2015; Rajendran et al., 2015). D-Ribose inhibited the transcription of tuf, pgm, fab, gap, and rpoN to about 0.39-fold, 0.56-fold, 0.20-fold, 0.27 -fold, and 0.78 -fold, respectively, while it increased the transcription of $n f o$ and rib to about 2.57 -fold and 4.87 -fold, respectively.

D-Ribose produced inhibition of AI-2 activity and biofilm formation of L-ZS9, which was induced by AI-2. Thus, the effect of AI-2 on gene expression was investigated. As shown in Figure 8, exogenous AI-2 changed the transcription of tuf, fba, gap, pgm, nfo, rib, and rpoN in L. paraplantarum L-ZS9. Opposite to the effect of D-ribose, AI-2 increased transcription of tuf, pgm, fab, gap and rpoN to about 30-fold, 20-fold, 11-fold, 10-fold, and 378-fold, respectively. And AI-2 decreased the 
TABLE 1 | Functional classification of differentially expressed proteins in cells of L. paraplantarum L-ZS9 treated with or without D-ribose.

\begin{tabular}{|c|c|c|c|c|c|c|}
\hline Spot no. & Accession no. & Gene & Protein name & Protein MW & Protein pl & Fold change $^{a}$ \\
\hline \multicolumn{7}{|c|}{ Carbon metabolism and carbohydrate metabolism } \\
\hline 2719 & gi| 380033724 & pgm & Phosphoglycerate mutase family protein & 26071.3 & 4.94 & 0.56 \\
\hline 3510 & gil 545603975 & rib & Ribokinase & 32080.3 & 5.02 & 3.24 \\
\hline 3612 & gi| 545603975 & rib & Ribokinase & 32080.3 & 5.02 & 3.01 \\
\hline 4613 & gi| 380031375 & fba & Fructose-bisphosphate aldolase & 30901.6 & 5.07 & 0.47 \\
\hline 5618 & gil 254555821 & gap & Glyceraldehyde-3-phosphate dehydrogenase & 36415.6 & 5.3 & 0.54 \\
\hline 5613 & gi| 380031375 & fba & Fructose-bisphosphate aldolase & 30901.6 & 5.07 & 2.34 \\
\hline 3206 & gi| 545606863 & ptk & Phosphoketolase & 88690.1 & 5.05 & 5.12 \\
\hline \multicolumn{7}{|c|}{ Amino acid metabolism } \\
\hline 6210 & gil 545603889 & $\operatorname{asn} B$ & Asparagine synthase & 73077.3 & 5.72 & 0.21 \\
\hline 6420 & gil 545606144 & murD & UDP-N-acetylmuramoyl-L-alanyl-D-glutamate synthetase & 49993.6 & 5.75 & 1.67 \\
\hline \multicolumn{7}{|c|}{ Nucleotide transport and metabolism } \\
\hline 3828 & gi| 254556519 & gmk & Guanylate kinase & 23445.7 & 4.99 & 2.33 \\
\hline 1613 & gil 545605966 & rihC & Ribonucleoside hydrolase RihC & 33030.6 & 4.67 & 2.18 \\
\hline 3516 & gil 545605039 & guaC & Guanosine $5^{\prime}$-monophosphate oxidoreductase & 39843.3 & 5.21 & 0.3 \\
\hline 4413 & gi| 544928185 & purA & Adenylosuccinate synthetase & 47207.3 & 5.25 & 0.46 \\
\hline 6615 & gil 545604536 & nfo & Endonuclease IV & 31603.1 & 5.65 & 2.1 \\
\hline \multicolumn{7}{|c|}{ Amino acid metabolism } \\
\hline 4615 & gi| 545605392 & smp & S-malonyltransferase & 33242.1 & 5.59 & 1.77 \\
\hline 5211 & gil 513840005 & lai & Linoleic acid isomerase & 64204.6 & 5.37 & 1.74 \\
\hline \multicolumn{7}{|c|}{ Transcription and translation } \\
\hline 2116 & gi| 545606085 & tuf & Elongation factor Tu & 43364.1 & 4.95 & 0.1 \\
\hline 3002 & gil 550695603 & rpoB & DNA-directed RNA polymerase subunit beta & 131576.3 & 4.87 & 0.02 \\
\hline 3207 & gil 545606497 & fusA & Elongation factor $\mathrm{G}$ & 76921.7 & 4.82 & 9.91 \\
\hline 3227 & gil 254556435 & thrs & Threonyl-tRNA synthetase & 73773 & 5.1 & 0.43 \\
\hline 3408 & gi| 545606490 & sers & Seryl-tRNA synthetase & 48103.2 & 5.15 & 2.2 \\
\hline 5801 & gi| 545605166 & $r p o N$ & Sigma 54 factor, RpoN & 21724.2 & 5.47 & 0.14 \\
\hline 5806 & gil 545605166 & $r p o N$ & Sigma 54 factor, RpoN & 21724.2 & 5.47 & 0.03 \\
\hline \multicolumn{7}{|l|}{ Other } \\
\hline 3714 & gi| 254556461 & $\operatorname{rrg}$ & Response regulator & 26356 & 5.06 & 0.55 \\
\hline 4235 & gil 545605165 & $\sec A$ & Preprotein translocase subunit SecA & 89545.2 & 5.16 & 3.13 \\
\hline 5401 & gi| 545603952 & nirD & Assimilatory nitrite reductase, subunit & 44407.8 & 5.51 & 2.2 \\
\hline 8604 & gil 545604158 & $a d h$ & Aryl-alcohol dehydrogenase family enzyme & 36955.8 & 6.71 & 2.03 \\
\hline
\end{tabular}

${ }^{\mathrm{a}} \mathrm{D}$-Ribose-treated vs. untreated cells.

transcription of gene $n f o$ and rib to about 0.21 -fold and 0.5 -fold, respectively. These results showed that AI-2 and D-ribose had opposite effects on the expression of $t u f, f b a, g a p, p g m, n f o, r i b$, and $r p o N$.

\section{tuf, fba, gap, pgm, nfo, rib, and rpoN Regulated Biofilm Formation of L. paraplantarum L-ZS9}

To validate the effects of tuf, fba, gap, pgm, nfo, rib, and rpoN gene expression on biofilm formation of L. paraplantarum L-ZS9, recombinant strains over-expressing these genes were constructed and their biofilm formation abilities were compared with those of the wild-type strain. Over-expression of these genes had no significant effect on the growth of $L$. paraplantarum L-ZS9 (Figure 9A). As shown in Figure 9B, over-expression of tuf, pgm, fba, gap, and $r p o N$ increased biofilm formation and over-expression of $n f o$ and rib inhibited biofilm formation of L. paraplantarum L-ZS9 significantly.

\section{DISCUSSION}

The LuxS/AI-2 QS system has been found in a variety of bacteria, including both Gram-negative and Gram-positive species. The non-species-specific signal AI-2, which acts as a universal QS signal for interaction between bacterial species, is a byproduct of SAM metabolism with LuxS as the key enzyme. Biosynthesis of AI-2 involves a three-step reaction, which is part of the methionine catabolism cycle. The first step is the removal of a methyl group from SAM, which is catalyzed by SAM-dependent methyltransferases. The resulting product, $\mathrm{SAH}$, is converted to $S$-ribosylhomocysteine by the enzyme Pfs (Parveen and Cornell, 2011). S-ribosylhomocysteine is hydrolyzed to 4,5-dihydroxy-2,3-pentanedione by LuxS (Schauder et al., 2001). 4,5-Dihydroxy-2,3-pentanedione is further auto-hydrolyzed to form AI-2. SAH exists widely in organisms and is transformed to homocysteine through one-step or two-step conversion. In most bacteria, SAH is metabolized using two-step conversion by the enzymes Pfs and LuxS with 


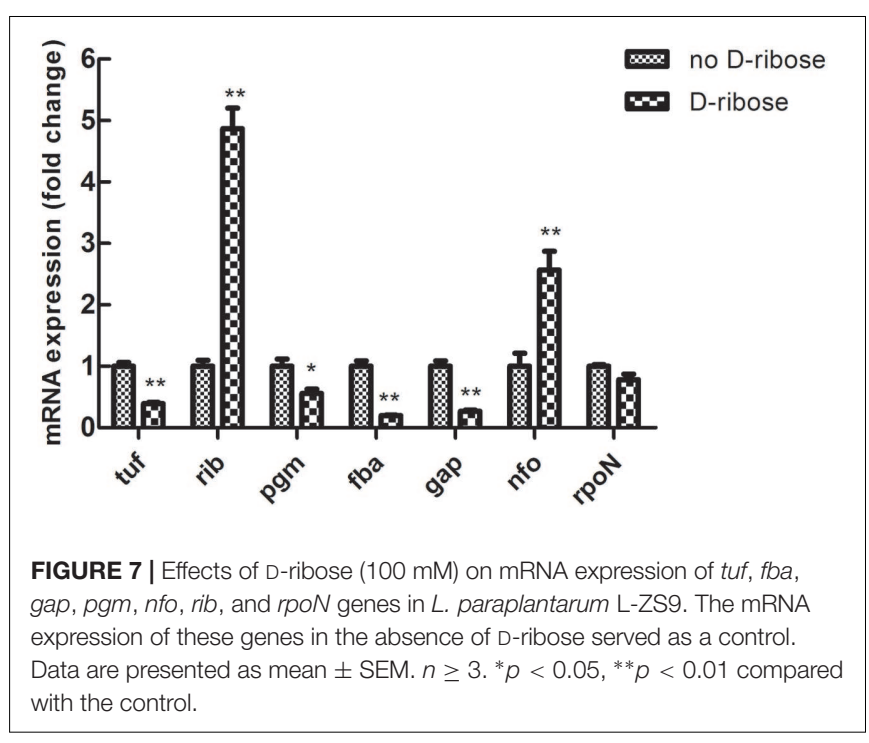

concomitant AI-2 production. However, in some prokaryotes and eukaryotes, SAH can be metabolized by one-step conversion using SAH hydrolase $(\mathrm{SahH})$ with no AI-2 production. In this study, genome sequence analysis suggested that L-ZS9 degrades SAH via the enzymes Pfs (MTAN) and LuxS to produce AI-2, without SAH hydrolase (SahH) (Figure 1). In addition, AI-2 activity in CFs of L-ZS9 was determined. AI2 activity is usually detected by using the reporter strain $V$. harveyi $\mathrm{BB} 170$ (sensor ${ }^{1-}$ sensor $^{2+}$ ), which only recognizes AI-2 (Vilchez et al., 2007). However, a high concentration of glucose and acidic $\mathrm{pH}$ can inhibit AI-2 activity (DeKeersmaecker and Vanderleyden, 2003; Turovskiy and Chikindas, 2006; Cuadra et al., 2016). Therefore, as for lactic acid bacteria, AI-2 activity detection is difficult under traditional cultural conditions and CF preparation. As a consequence, there was no AI-2 activity detected in L. paraplantarum L-ZS9 when the strain was cultured in MRS broth (data not shown). However, AI-2 activity was detected when L. paraplantarum L-ZS9 was cultured in skim milk medium and CFs were adjusted to $\mathrm{pH} 7.0$ (Figure 2). This preparation method can also be used for AI-2 activity detection of other lactic acid bacteria. According to these results, L. paraplantarum L-ZS9 may have the capacity to communicate with itself and other strains by AI-2. Therefore, LuxS/AI-2 QSI could play an important role in interfering with the social behavior of L-ZS9.

Ribose has been reported to be used as a QSI of the LuxS/AI-2 QS system by competing for AI-2 receptor (James et al., 2006; Armbruster et al., 2011). Our data showed that D-ribose could inhibit AI-2 activity of L-ZS9, suggesting that it can be used as a QSI of the LuxS/AI-2 QS system in L-ZS9. LuxS/AI-2 QS regulates biofilm formation of many bacteria such as Streptococcus mitis, Pseudomonas aeruginosa PAO1, Eikenella corrodens, Candida albicans, Riemerella anatipestifer, Aggregatibacter actinomycetemcomitans, and Bifidobacteria (Shao et al., 2007; Karim et al., 2013; Bachtiar et al., 2014; Christiaen et al., 2014; Han et al., 2015; Li H. et al., 2015; Wang et al., 2016). However, limited studies have been conducted on the regulation of biofilm formation in Lactobacillus by the AI-2/LuxS QS system. It has been reported that luxS of Lactobacillus rhamnosus GG plays a central metabolic role in biofilm formation (Lebeer et al., 2007a). Lactobacillus uses the AI-2 signal to respond to environment stress and to regulate growth and metabolism (Lebeer et al., 2007b, 2008; Moslehi-Jenabian et al., 2009; Yeo et al., 2015). The present study showed that AI-2 increased biofilm formation of $L$. paraplantarum L-ZS9 (Figure 5A). D-Ribose has been reported to inhibit co-culture biofilm formation of $P$. aeruginosa PAO1 and Streptococcus mitis significantly (Wang et al., 2016). Ribose could also inhibit streptococcal biofilm formation (Lee et al., 2015). In this study, D-ribose was confirmed to significantly inhibit the biofilm formation of L. paraplantarum L-ZS9 as well (Figure 5B). These results suggest that a universal QSI such as ribose could inhibit biofilm formation of non-pathogenic and beneficial microorganisms, which must be taken into consideration when a universal QSI is used for against pathogens.

To investigate the inhibition mechanism of $\mathrm{D}$-ribose in regulating biofilm formation, relevant genes were firstly identified by comparative proteomic analysis. According to the proteomic analysis and previous research (Wolfe et al., 2004; Da et al., 2008; Kiedrowski et al., 2011; Iyer and Hancock, 2012; Sahu et al., 2012; Zhou et al., 2012; Hao et al., 2013; Li H. et al., 2015; Rajendran et al., 2015), D-ribose may function through gene regulation as shown in Figure 10. The influence of Dribose and AI-2 on gene transcription (tuf, fba, gap, pgm, $n f o$, rib, and $r p o N)$ was then further evaluated by qRTPCR. Our data showed that D-ribose, a LuxS/AI-2 QS system QSI, regulated the expression of tuf, fba, gap, pgm, nfo, rib, and rpoN in an opposite fashion to AI-2. Biofilm assays of recombinant strains further indicated that the overexpression of $f b a, g a p$, and pgm could increase the biofilm formation of L-ZS9. The fba, gap, and pgm genes play key roles in the glycolytic pathway (EMP), which is important for energy metabolism. $f b a$ is responsible for transformation of frucose-1,6-diphosphate to dihydroxy acetone phosphate, which is transformed to 1,3-diphosphoglycerate by gap, and pgm transforms 3-phosphoglyceride to 2-phosphoglycerate. EMP has been reported to play key roles in biofilm formation and procession of Saccharomyces cerevisiae (Li H. et al., 2015). Proteins involved in EMP were indicated to be up-regulated in biofilm cells of Listeria monocytogenes compared with planktonic cells (Zhou et al., 2012). Our data also showed that EMP in L-ZS9 was positively correlated with biofilm formation. In addition, EMP may play roles in sugar metabolism as well. Previous studies elucidated that extracellular polysaccharide (EPS) is important in biofilm formation (Blackledge et al., 2013). Stoodley et al. (2002) reported the possible role of QS as a signal transduction system to initiate the production of alginate and possibly other types of EPS in $P$. aeruginosa. Based on these studies, it is logical to deduce that AI-2 and D-ribose could affect biofilm formation of L. paraplantarum L-ZS9 by regulating EPS synthesis. 


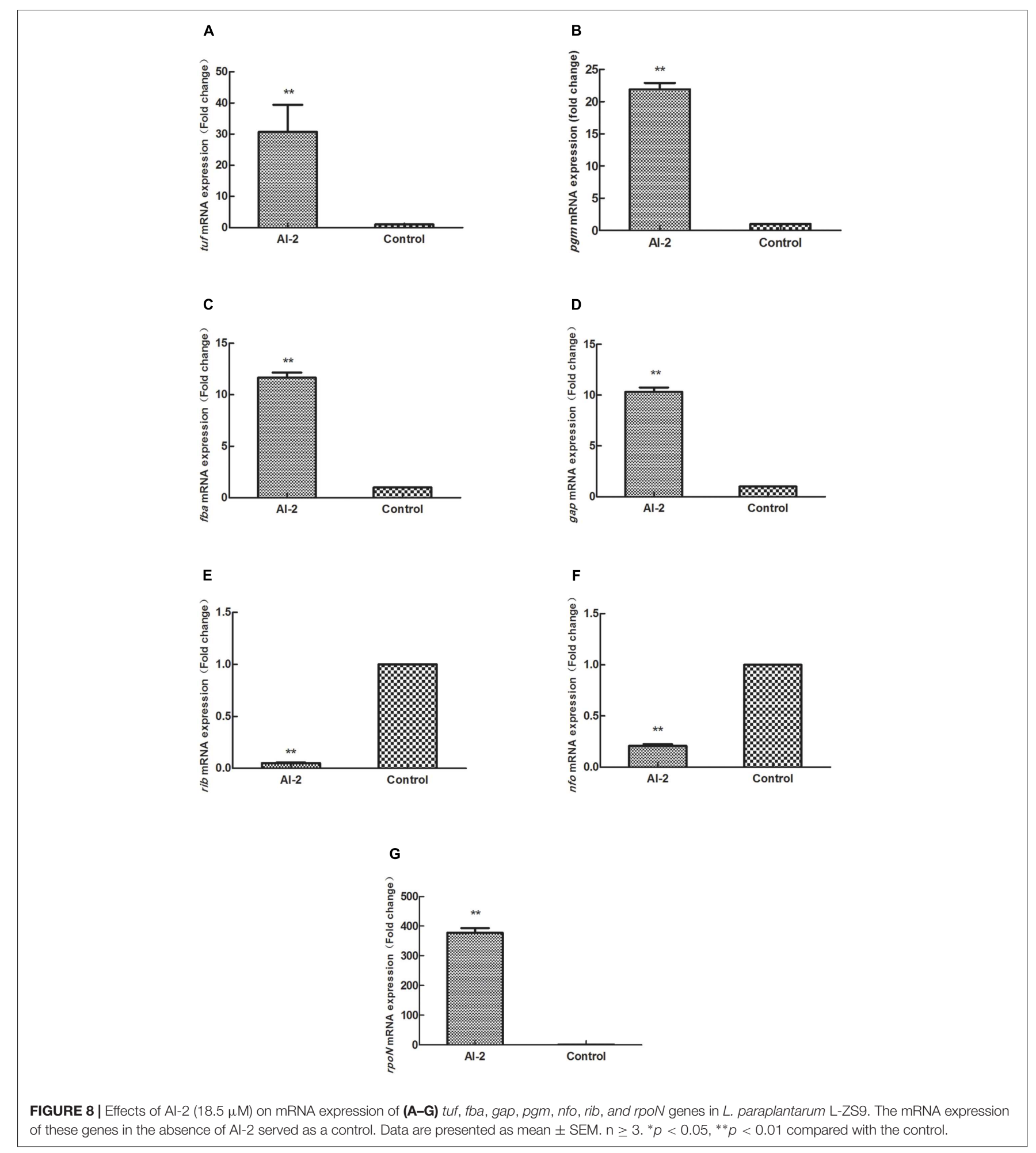

D-Ribose and AI-2 increased and decreased the expression of the $n f o$ gene, respectively, which encodes an endonuclease. Extracellular DNA is one of the important components of biofilm matrix, which could be degraded by various nucleases. DNase is able to break biofilm formation of Gram-positive and Gram-negative bacteria rapidly (Nijland et al., 2010). It was reported that $c o m E B$ regulated biofilm formation of Staphylococcus lugdunensis by influencing production of DNA (Rajendran et al., 2015). As for Acinetobacter baumannii AIIMS 7, extracellular DNA was degraded and biofilm was decreased 

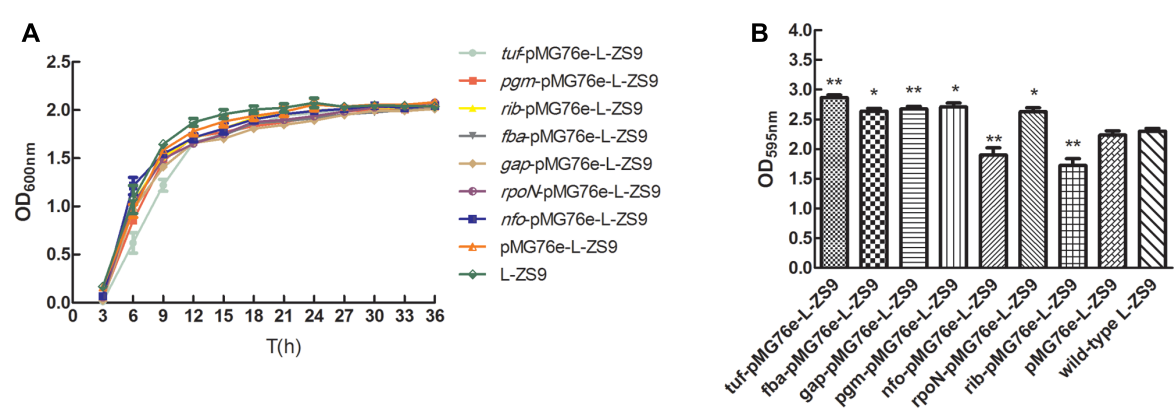

FIGURE 9 | Growth curves (A) and biofilm formation (B) of recombinant L. paraplantarum L-ZS9 strains. The wild-type strain L. paraplantarum L-ZS9 served as a control. Data are presented as mean \pm SEM. $n \geq 3 .{ }^{*} p<0.05,{ }^{* *} p<0.01$ compared with the control.

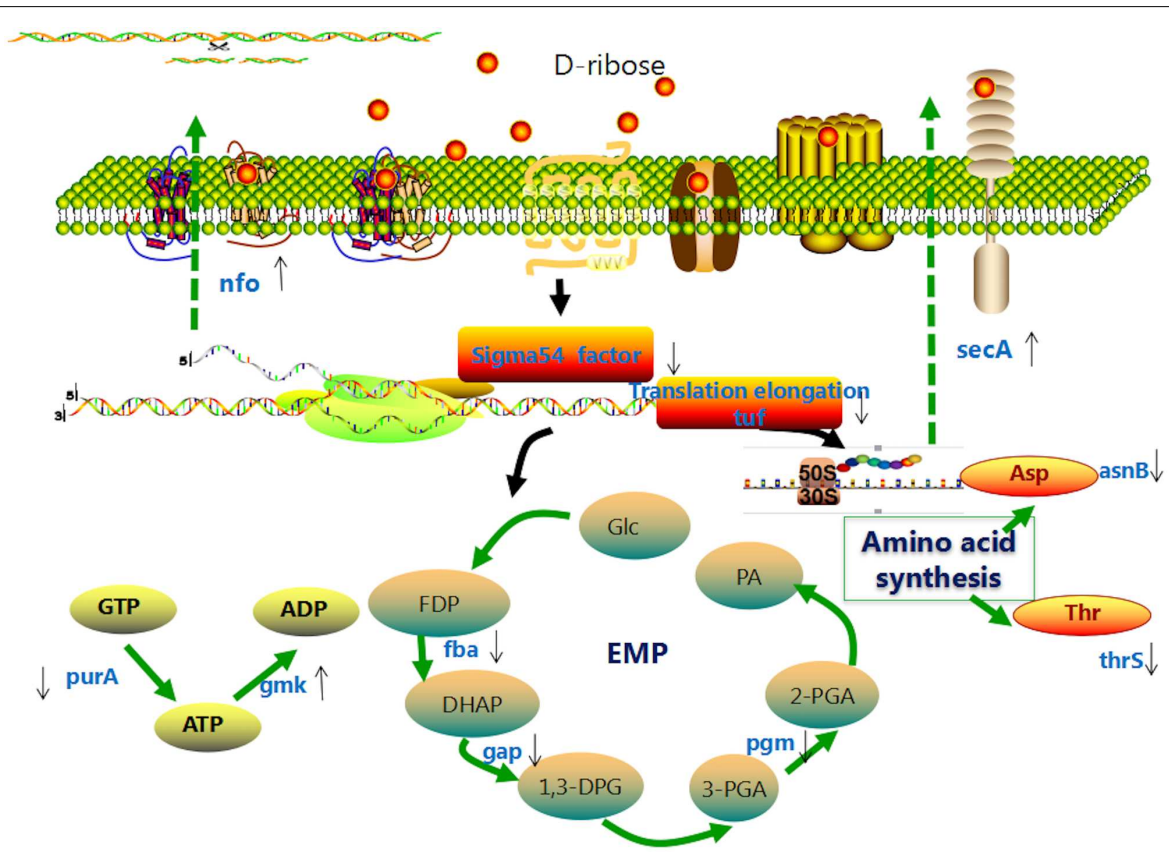

FIGURE 10 | Hypothetical model of the effect of D-ribose on L. paraplantarum L-ZS9 cells. Glc, glucose; FDP, fructose-1,6-diphosphate; DHAP, dihydroxyacetone phosphate; 1,3-DPG, 1,3-diphosphoglyceric acid; 3-PGA, 3-phosphoglyceric acid; 2-PGA, 2-phosphoglyceric acid; PA, pyruvic acid; EMP, glycolysis.

to $59.41 \%$ after treatment with DNase I (Sahu et al., 2012). In Staphylococcus aureus and Neisseria gonorrhoeae, nuclease modulated biofilm formation by regulating extracellular DNA (Kiedrowski et al., 2011; Steichen et al., 2011). Expression of extracellular endonucleases also influenced the biofilm formation of Shewanella oneidensis MR-1 (Godeke et al., 2011), and DNase 1L2 suppressed biofilm formation of $P$. aeruginosa and Staphylococcus aureus (Eckhart et al., 2007). Similar to other research, over-expression of endonuclease also inhibited biofilm formation of L-ZS9 in our study.

Our data also showed that the biofilm formation of strain tuf-76e-L-ZS9 was increased compared with the wild-type strain. The tuf gene encodes elongation factor thermo unstable (EF-Tu) and functions in translation elongation, protein folding, and protection from stress (Caldas et al., 1998). Although no research has reported that EF-Tu modulates biofilm formation directly, it has been identified as an adherence-related factor that may affect biofilm formation. EF-Tu plays important roles in adherence of Lactobacillus, such as Lactobacillus acidophilus ATCC 4356, Lactobacillus plantarum 423, and Lactobacillus johnsonii NCC533 (La1) (Ramiah et al., 2008, 2009; Dhanani and Bagchi, 2013). This is the first time to our knowledge that EF-Tu has been reported to modulate biofilm formation directly, which may be due to regulation of the initial adherence of L-ZS9. Over-expression of rpoN that encodes $\sigma 54$ factor also promoted the biofilm formation of L-ZS9. The $\sigma 54$ factor is structurally and functionally distinct from all other $\sigma$ factors and is required for initiation of transcription (Shingler, 2011). In addition, $\sigma 54$ regulates utilization of alternative carbon sources, detoxification systems, assembly of motility organs, and production of extracellular 
alginate (Maxson and Darwin, 2006). Although the role of $\sigma 54$ remains unclear in Lactobacillus, it has been reported that $\sigma 54$ correlates with biofilm formation directly or indirectly in other bacteria (Belik et al., 2008; Saldias et al., 2008; Iyer and Hancock, 2012; Hao et al., 2013). For example, o54 controls motility, biofilm formation, and colonization in Vibrio fischeri (Wolfe et al., 2004; Yip et al., 2005) and regulates genes involved in type I and type IV pili biogenesis to influence the biofilm formation of Xylella fastidiosa (Da et al., 2008). Based on the results of these studies, $\sigma 54$ may play similar roles to regulate biofilm formation of L-ZS9. In addition, it was identified that rib also regulated biofilm formation of L-ZS9, and the underlying mechanism still needs to be further investigated. By exploring the effects of D-ribose on L-ZS9, this study identified the importance of serious consideration of LuxS/AI-2 QSI application due to its effects on non-pathogenic or beneficial bacteria. Modification of specific genes (for example genes identified in this research) should be conducted in order to protect these organisms from the QSI.

\section{CONCLUSION}

Lactobacillus paraplantarum L-ZS9 contains key genes related to the LuxS/AI-2 QS system and is able to produce AI-2. D-Ribose can be used as a QSI of L-ZS9 and inhibits biofilm formation of

\section{REFERENCES}

Armbruster, C. E., Pang, B., Murrah, K., Juneau, R. A., Perez, A. C., Weimer, K. E., et al. (2011). RbsB (NTHI_0632) mediates quorum signal uptake in nontypeable Haemophilus influenzae strain 86-028NP. Mol. Microbiol. 82, 836-850. doi: 10.1111/j.1365-2958.2011.07831.x

Bachtiar, E. W., Bachtiar, B. M., Jarosz, L. M., Amir, L. R., Sunarto, H., Ganin, H., et al. (2014). AI-2 of Aggregatibacter actinomycetemcomitans inhibits Candida albicans biofilm formation. Front. Cell. Infect. Microbiol. 4:94. doi: 10.3389/ fcimb.2014.00094

Bassler, B. L., Wright, M., Showalter, R. E., and Silverman, M. R. (1993). Intercellular signalling in Vibrio harveyi: sequence and function of genes regulating expression of luminescence. Mol. Microbiol. 9, 773-786. doi: 10.1111/ j.1365-2958.1993.tb01737.x

Belik, A. S., Tarasova, N. N., and Khmel', I. A. (2008). Regulation of biofilm formation in Escherichia coli K12: effect of mutations in HNS, StpA, lon, and rpoN genes. Mol. Gen. Mikrobiol. Virusol. 23, 159-162. doi: 10.3103/ S0891416808040010

Bixler, G. D., and Bhushan, B. (2012). Biofouling: lessons from nature. Philos. Trans. A Math. Phys. Eng. Sci. 370, 2381-2417. doi: 10.1098/rsta.2011.0502

Blackledge, M. S., Worthington, R. J., and Melander, C. (2013). Biologically inspired strategies for combating bacterial biofilms. Curr. Opin. Pharmacol. 13, 699-706. doi: 10.1016/j.coph.2013.07.004

Brackman, G., Celen, S., Baruah, K., Bossier, P., Van Calenbergh, S., Nelis, H. J., et al. (2009). AI-2 quorum-sensing inhibitors affect the starvation response and reduce virulence in several Vibrio species, most likely by inferring with LuxPQ. Microbiology 155, 4114-4122. doi: 10.1099/mic.0.032474-0

Brackman, G., Celen, S., Hillaert, U., Van Calenbergh, S., Cos, P., Maes, L., et al. (2011). Structure-activity relationship of cinnamaldehyde analogs as inhibitors of AI-2 based quorum sensing and their effect on virulence of Vibrio spp. PLOS ONE 6:e16084. doi: 10.1371/journal.pone.0016084

Buck, B. L., Azcarate-Peril, M. A., and Klaenhammer, T. R. (2009). Role of autoinducer-2 on the adhesion ability of Lactobacillus acidophilus. J. Appl. Microbiol. 107, 269-279. doi: 10.1111/j.1365-2672.2009.04204.x

Caldas, T. D., El, Y. A., and Richarme, G. (1998). Chaperone properties of bacterial elongation factor EF-Tu. J. Biol. Chem. 273, 11478-11482. doi: 10.1074/jbc.273. 19.11478
L-ZS9 in contrast to exogenous AI-2 by regulating expression of the tuf, fba, gap, pgm, nfo, rib, and rpoN genes. This research provides new information about regulation mechanisms of the LuxS/AI-2 QS system in Lactobacillus and will be useful for determining the reasonable application of QSI.

\section{AUTHOR CONTRIBUTIONS}

LL and PL designed the experiments. LL, RW, and JZ performed the experiments. LL, NS, and PL analyzed the results and wrote the manuscript.

\section{FUNDING}

This work was supported by grants from the National Natural Science Foundation of China (31671831 and 31471707).

\section{SUPPLEMENTARY MATERIAL}

The Supplementary Material for this article can be found online at: http://journal.frontiersin.org/article/10.3389/fmicb. 2017.01860/full\#supplementary-material

Cao, J. G., and Meighen, E. A. (1989). Purification and structural identification of an autoinducer for the luminescence system of Vibrio harveyi. J. Biol. Chem. 264, 21670-21676.

Cho, Y. J., Song, H. Y., Ben, A. H., Choi, B. K., Eunju, R., Cho, Y. A., et al. (2016). In vivo inhibition of Porphyromonas gingivalis growth and prevention of periodontitis with quorum-sensing inhibitors. J. Periodontol. 87, 1075-1082. doi: 10.1902/jop.2016.160070

Christiaen, S. E., O’Connell, M. M., Bottacini, F., Lanigan, N., Casey, P. G., Huys, G., et al. (2014). Autoinducer-2 plays a crucial role in gut colonization and probiotic functionality of Bifidobacterium breve UCC2003. PLOS ONE 9:e98111. doi: 10.1371/journal.pone.0098111

Cuadra, G. A., Frantellizzi, A. J., Gaesser, K. M., Tammariello, S. P., and Ahmed, A. (2016). Autoinducer-2 detection among commensal oral streptococci is dependent on pH and boric acid. J. Microbiol. 54, 492-502. doi: 10.1007/s12275016-5507-z

Da, S. N. J., Koide, T., Abe, C. M., Gomes, S. L., and Marques, M. V. (2008). Role of sigma54 in the regulation of genes involved in type I and type IV pili biogenesis in Xylella fastidiosa. Arch. Microbiol. 189, 249-261. doi: 10.1007/s00203-0070314-x

DeKeersmaecker, S. C., and Vanderleyden, J. (2003). Constraints on detection of autoinducer-2 (AI-2) signalling molecules using Vibrio harveyi as a reporter. Microbiology 149, 1953-1956. doi: 10.1099/mic.0.C0117-0

Dhanani, A. S., and Bagchi, T. (2013). The expression of adhesin EF-Tu in response to mucin and its role in Lactobacillus adhesion and competitive inhibition of enteropathogens to mucin. J. Appl. Microbiol. 115, 546-554. doi: 10.1111/jam. 12249

Drescher, K., Shen, Y., Bassler, B. L., and Stone, H. A. (2013). Biofilm streamers cause catastrophic disruption of flow with consequences for environmental and medical systems. Proc. Natl. Acad. Sci. U.S.A. 110, 4345-4350. doi: 10.1073/ pnas. 1300321110

Eckhart, L., Fischer, H., Barken, K. B., Tolker-Nielsen, T., and Tschachler, E. (2007). DNase1L2 suppresses biofilm formation by Pseudomonas aeruginosa and Staphylococcus aureus. Br. J. Dermatol. 156, 1342-1345. doi: 10.1111/j. 1365-2133.2007.07886.x

Godeke, J., Heun, M., Bubendorfer, S., Paul, K., and Thormann, K. M. (2011). Roles of two Shewanella oneidensis MR-1 extracellular endonucleases. Appl. Environ. Microbiol. 77, 5342-5351. doi: 10.1128/AEM.00643-11 
Gospodarek, E., Bogiel, T., and Zalas-Wiecek, P. (2009). Communication between microorganisms as a basis for production of virulence factors. Pol. J. Microbiol. 58, 191-198.

Han, X., Liu, L., Fan, G., Zhang, Y., Xu, D., Zuo, J., et al. (2015). Riemerella anatipestifer lacks luxS, but can uptake exogenous autoinducer-2 to regulate biofilm formation. Res. Microbiol. 166, 486-493. doi: 10.1016/j.resmic.2015. 06.004

Hao, B., Mo, Z. L., Xiao, P., Pan, H. J., Lan, X., and Li, G. Y. (2013). Role of alternative sigma factor 54 (RpoN) from Vibrio anguillarum M3 in protease secretion, exopolysaccharide production, biofilm formation, and virulence. Appl. Microbiol. Biotechnol. 97, 2575-2585. doi: 10.1007/s00253-0124372-x

Harjai, K., Gupta, R. K., and Sehgal, H. (2014). Attenuation of quorum sensing controlled virulence of Pseudomonas aeruginosa by cranberry. Indian J. Med. Res. 139, 446-453.

Hoiby, N., Bjarnsholt, T., Givskov, M., Molin, S., and Ciofu, O. (2010). Antibiotic resistance of bacterial biofilms. Int. J. Antimicrob. Agents 35, 322-332. doi: 10.1016/j.ijantimicag.2009.12.011

Iyer, V. S., and Hancock, L. E. (2012). Deletion of sigma(54) (rpoN) alters the rate of autolysis and biofilm formation in Enterococcus faecalis. J. Bacteriol. 194, 368-375. doi: 10.1128/JB.06046-11

James, D., Shao, H., Lamont, R. J., and Demuth, D. R. (2006). The Actinobacillus actinomycetemcomitans ribose binding protein $\mathrm{RbsB}$ interacts with cognate and heterologous autoinducer 2 signals. Infect. Immun. 74, 4021-4029. doi: 10.1128/ IAI.01741-05

Karim, M. M., Hisamoto, T., Matsunaga, T., Asahi, Y., Noiri, Y., Ebisu, S., et al. (2013). LuxS affects biofilm maturation and detachment of the periodontopathogenic bacterium Eikenella corrodens. J. Biosci. Bioeng. 116, 313-318. doi: 10.1016/j.jbiosc.2013.03.013

Kiedrowski, M. R., Kavanaugh, J. S., Malone, C. L., Mootz, J. M., Voyich, J. M., Smeltzer, M. S., et al. (2011). Nuclease modulates biofilm formation in community-associated methicillin-resistant Staphylococcus aureus. PLOS ONE 6:e26714. doi: 10.1371/journal.pone.0026714

Lakshman, D. K., Natarajan, S. S., Lakshman, S., Garrett, W. M., and Dhar, A. K. (2008). Optimized protein extraction methods for proteomic analysis of Rhizoctonia solani. Mycologia 100, 867-875. doi: 10.3852/08-065

Lebeer, S., Claes, I. J., Verhoeven, T. L., Shen, C., Lambrichts, I., Ceuppens, J. L., et al. (2008). Impact of luxS and suppressor mutations on the gastrointestinal transit of Lactobacillus rhamnosus GG. Appl. Environ. Microbiol. 74, 4711-4718. doi: 10.1128/AEM.00133-08

Lebeer, S., De Keersmaecker, S. C., Verhoeven, T. L., Fadda, A. A., Marchal, K., and Vanderleyden, J. (2007a). Functional analysis of luxS in the probiotic strain Lactobacillus rhamnosus GG reveals a central metabolic role important for growth and biofilm formation. J. Bacteriol. 189, 860-871. doi: 10.1128/JB. 01394-06

Lebeer, S., Verhoeven, T. L., Perea, V. M., Vanderleyden, J., and De Keersmaecker, S. C. (2007b). Impact of environmental and genetic factors on biofilm formation by the probiotic strain Lactobacillus rhamnosus GG. Appl. Environ. Microbiol. 73, 6768-6775. doi: 10.1128/AEM.01393-07

Lee, H. J., Kim, S. C., Kim, J., Do, A., Han, S. Y., Lee, B. D., et al. (2015). Synergistic inhibition of streptococcal biofilm by ribose and xylitol. Arch. Oral Biol. 60, 304-312. doi: 10.1016/j.archoralbio.2014.11.004

Lepargneur, J. P., and Rousseau, V. (2002). Protective role of the Doderlein flora. J. Gynecol. Obstet. Biol. Reprod. 31, 485-494.

Li, H., Li, X., Wang, Z., Fu, Y., Ai, Q., Dong, Y., et al. (2015). Autoinducer2 regulates Pseudomonas aeruginosa PAO1 biofilm formation and virulence production in a dose-dependent manner. BMC Microbiol. 15:192. doi: 10.1186/ s12866-015-0529-y

Li, Z., Chen, Y., Liu, D., Zhao, N., Cheng, H., Ren, H., et al. (2015). Involvement of glycolysis/gluconeogenesis and signaling regulatory pathways in Saccharomyces cerevisiae biofilms during fermentation. Front. Microbiol. 6:139. doi: 10.3389/ fmicb.2015.00139

Liu, L., and Li, P. (2016). Complete genome sequence of Lactobacillus paraplantarum L-ZS9, a probiotic starter producing class II bacteriocins. J. Biotechnol. 222, 15-16. doi: 10.1016/j.jbiotec.2016.02.003

Livak, K. J., and Schmittgen, T. D. (2001). Analysis of relative gene expression data using real-time quantitative PCR and the $2^{-\Delta \Delta C_{\mathrm{T}}}$ method. Methods 25, 402-408. doi: 10.1006/meth.2001.1262
Maxson, M. E., and Darwin, A. J. (2006). Multiple promoters control expression of the Yersinia enterocolitica phage-shock-protein a (PspA) operon. Microbiology 152, 1001-1010. doi: 10.1099/mic.0.28714-0

Moslehi-Jenabian, S., Gori, K., and Jespersen, L. (2009). AI-2 signalling is induced by acidic shock in probiotic strains of Lactobacillus spp. Int. J. Food Microbiol. 135, 295-302. doi: 10.1016/j.ijfoodmicro.2009.08.011

Nadell, C. D., Drescher, K., and Foster, K. R. (2016). Spatial structure, cooperation and competition in biofilms. Nat. Rev. Microbiol. 14, 589-600. doi: 10.1038/ nrmicro. 2016.84

Nijland, R., Hall, M. J., and Burgess, J. G. (2010). Dispersal of biofilms by secreted, matrix degrading, bacterial DNase. PLOS ONE 5:e15668. doi: 10.1371/journal. pone.0015668

Olsen, I. (2015). Biofilm-specific antibiotic tolerance and resistance. Eur. J. Clin. Microbiol. Infect. Dis. 34, 877-886. doi: 10.1007/s10096-015-2323-z

Pan, J., and Ren, D. (2009). Quorum sensing inhibitors: a patent overview. Expert Opin. Ther. Pat. 19, 1581-1601. doi: 10.1517/13543770903222293

Park, H., Shin, H., Lee, K., and Holzapfel, W. (2016). Autoinducer-2 properties of kimchi are associated with lactic acid bacteria involved in its fermentation. Int. J. Food Microbiol. 225, 38-42. doi: 10.1016/j.ijfoodmicro.2016.03.007

Parveen, N., and Cornell, K. A. (2011). Methylthioadenosine/Sadenosylhomocysteine nucleosidase, a critical enzyme for bacterial metabolism. Mol. Microbiol. 79, 7-20. doi: 10.1111/j.1365-2958.2010.07455.x

Penesyan, A., Gillings, M., and Paulsen, I. T. (2015). Antibiotic discovery: combatting bacterial resistance in cells and in biofilm communities. Molecules 20, 5286-5298. doi: 10.3390/molecules20045286

Rajendran, N. B., Eikmeier, J., Becker, K., Hussain, M., Peters, G., and Heilmann, C. (2015). Important contribution of the novel locus comEB to extracellular DNAdependent Staphylococcus lugdunensis biofilm formation. Infect. Immun. 83, 4682-4692. doi: 10.1128/IAI.00775-15

Ramiah, K., van Reenen, C. A., and Dicks, L. M. (2008). Surface-bound proteins of Lactobacillus plantarum 423 that contribute to adhesion of Caco- 2 cells and their role in competitive exclusion and displacement of Clostridium sporogenes and Enterococcus faecalis. Res. Microbiol. 159, 470-475. doi: 10.1016/j.resmic. 2008.06.002

Ramiah, K., van Reenen, C. A., and Dicks, L. M. (2009). Expression of the mucus adhesion gene $m u b$, surface layer protein $s l p$ and adhesion-like factor EF-TU of Lactobacillus acidophilus ATCC 4356 under digestive stress conditions, as monitored with real-time PCR. Probiotics Antimicrob. Proteins 1, 91. doi: 10.1007/s12602-009-9009-8

Roy, V., Meyer, M. T., Smith, J. A., Gamby, S., Sintim, H. O., Ghodssi, R., et al. (2013). AI-2 analogs and antibiotics: a synergistic approach to reduce bacterial biofilms. Appl. Microbiol. Biotechnol. 97, 2627-2638. doi: 10.1007/s00253-0124404-6

Ryu, E. J., Sim, J., Sim, J., Lee, J., and Choi, B. K. (2016). D-Galactose as an autoinducer 2 inhibitor to control the biofilm formation of periodontopathogens. J. Microbiol. 54, 632-637. doi: 10.1007/s12275-0166345-8

Sahu, P. K., Iyer, P. S., Oak, A. M., Pardesi, K. R., and Chopade, B. A. (2012). Characterization of eDNA from the clinical strain Acinetobacter baumannii AIIMS 7 and its role in biofilm formation. ScientificWorldJournal 2012:973436. doi: 10.1100/2012/973436

Saldias, M. S., Lamothe, J., Wu, R., and Valvano, M. A. (2008). Burkholderia cenocepacia requires the RpoN sigma factor for biofilm formation and intracellular trafficking within macrophages. Infect. Immun. 76, 1059-1067. doi: 10.1128/IAI.01167-07

Schauder, S., Shokat, K., Surette, M. G., and Bassler, B. L. (2001). The LuxS family of bacterial autoinducers: biosynthesis of a novel quorum-sensing signal molecule. Mol. Microbiol. 41, 463-476. doi: 10.1046/j.1365-2958.2001.02532.x

Shao, H., Lamont, R. J., and Demuth, D. R. (2007). Autoinducer 2 is required for biofilm growth of Aggregatibacter (Actinobacillus) actinomycetemcomitans. Infect. Immun. 75, 4211-4218. doi: 10.1128/IAI.00402-07

Shingler, V. (2011). Signal sensory systems that impact sigma54-dependent transcription. FEMS Microbiol. Rev. 35, 425-440. doi: 10.1111/j.1574-6976. 2010.00255.x

Steichen, C. T., Cho, C., Shao, J. Q., and Apicella, M. A. (2011). The Neisseria gonorrhoeae biofilm matrix contains DNA, and an endogenous nuclease controls its incorporation. Infect. Immun. 79, 1504-1511. doi: 10.1128/IAI. 01162-10 
Stoodley, P., Sauer, K., Davies, D. G., and Costerton, J. W. (2002). Biofilms as complex differentiated communities. Annu. Rev. Microbiol. 56, 187-209. doi: 10.1146/annurev.micro.56.012302.160705

Sun, Z., He, X., Brancaccio, V. F., Yuan, J., and Riedel, C. U. (2014). Bifidobacteria exhibit LuxS-dependent autoinducer 2 activity and biofilm formation. PLOS ONE 9:e88260. doi: 10.1371/journal.pone.0088260

Taylor, P. K., Yeung, A. T., and Hancock, R. E. (2014). Antibiotic resistance in Pseudomonas aeruginosa biofilms: towards the development of novel antibiofilm therapies. J. Biotechnol. 191, 121-130. doi: 10.1016/j.jbiotec.2014. 09.003

Thompson, J. A., Oliveira, R. A., Djukovic, A., Ubeda, C., and Xavier, K. B. (2015). Manipulation of the quorum sensing signal AI-2 affects the antibiotic-treated gut microbiota. Cell Rep. 10, 1861-1871. doi: 10.1016/j.celrep.2015.02.049

Turovskiy, Y., and Chikindas, M. L. (2006). Autoinducer-2 bioassay is a qualitative, not quantitative method influenced by glucose. J. Microbiol. Methods 66, 497-503. doi: 10.1016/j.mimet.2006.02.001

Vilchez, R., Lemme, A., Thiel, V., Schulz, S., Sztajer, H., and Wagner-Dobler, I. (2007). Analysing traces of autoinducer-2 requires standardization of the Vibrio harveyi bioassay. Anal. Bioanal. Chem. 387, 489-496. doi: 10.1007/s00216-0060824-4

Wang, Y., Cheung, Y. H., Yang, Z., Chiu, J. F., Che, C. M., and He, Q. Y. (2006). Proteomic approach to study the cytotoxicity of dioscin (saponin). Proteomics 6, 2422-2432. doi: 10.1002/pmic.200500595

Wang, Y., Sun, Y., Zhang, X., Zhang, Z. C., Song, J. Y., Gui, M., et al. (2015). Bacteriocin-producing probiotics enhance the safety and functionality of sturgeon sausage. Food Control 50, 729-735. doi: 10.1016/j.foodcont.2014.09. 045

Wang, Z., Xiang, Q., Yang, T., Li, L., Yang, J., Li, H., et al. (2016). Autoinducer-2 of Streptococcus mitis as a target molecule to inhibit pathogenic multi-species biofilm formation in vitro and in an endotracheal intubation rat model. Front. Microbiol. 7:88. doi: 10.3389/fmicb.2016.00088
Wolfe, A. J., Millikan, D. S., Campbell, J. M., and Visick, K. L. (2004). Vibrio fischeri sigma54 controls motility, biofilm formation, luminescence, and colonization. Appl. Environ. Microbiol. 70, 2520-2524. doi: 10.1128/AEM.70.4.2520-2524. 2004

Yeo, S., Park, H., Ji, Y., Park, S., Yang, J., Lee, J., et al. (2015). Influence of gastrointestinal stress on autoinducer-2 activity of two Lactobacillus species. FEMS Microbiol. Ecol. 91:fiv065. doi: 10.1093/femsec/ fiv065

Yip, E. S., Grublesky, B. T., Hussa, E. A., and Visick, K. L. (2005). A novel, conserved cluster of genes promotes symbiotic colonization and sigma-dependent biofilm formation by Vibrio fischeri. Mol. Microbiol. 57, 1485-1498. doi: 10.1111/j.13652958.2005.04784.x

Zhang, X., Wang, Y., Liu, L., Wei, Y., Shang, N., Zhang, X., et al. (2016). Two-peptide bacteriocin PlnEF causes cell membrane damage to Lactobacillus plantarum. Biochim. Biophys. Acta 1858, 274-280. doi: 10.1016/j.bbamem.2015. 11.018

Zhou, Q., Feng, X., Zhang, Q., Feng, F., Yin, X., Shang, J., et al. (2012). Carbon catabolite control is important for Listeria monocytogenes biofilm formation in response to nutrient availability. Curr. Microbiol. 65, 35-43. doi: 10.1007/ s00284-012-0125-4

Conflict of Interest Statement: The authors declare that the research was conducted in the absence of any commercial or financial relationships that could be construed as a potential conflict of interest.

Copyright (c) $2017 \mathrm{Liu}, \mathrm{Wu}$, Zhang, Shang and Li. This is an open-access article distributed under the terms of the Creative Commons Attribution License (CC BY). The use, distribution or reproduction in other forums is permitted, provided the original author(s) or licensor are credited and that the original publication in this journal is cited, in accordance with accepted academic practice. No use, distribution or reproduction is permitted which does not comply with these terms. 